\title{
It's the adherence, stupid (that determines asthma control in preschool children)!
}

\author{
Ted Klok', Adrian A. Kaptein², Eric J. Duiverman ${ }^{3,4}$ and Paul L. Brand ${ }^{1,5}$ \\ Affiliations: 'Princess Amalia Children's Clinic, Isala Klinieken, Zwolle, ${ }^{2}$ Unit of Psychology, Leiden University \\ Medical Centre, Leiden, ${ }^{3}$ University of Groningen, University Medical Center Groningen, Beatrix Children's \\ Hospital, Dept of Paediatrics, Division of Paediatric Pulmonology, Groningen, "GRIAC Research Institute, \\ University Medical Centre Groningen, Groningen, and ${ }^{5}$ UMCG Postgraduate School of Medicine, University \\ Medical Centre Groningen, Groningen, The Netherlands.
}

Correspondence: T. Klok, Princess Amalia Children's Clinic, Isala Klinieken, Dr van Heesweg 2, 8025AB, Zwolle, The Netherlands. E-mail: contactQtedklok.nl

ABSTRACT Although guideline-based asthma care and adherence to inhaled corticosteroids are predictors of asthma control, the role of adherence in maintaining long-term asthma control is largely unknown. This study was designed to explore the relationship between adherence to inhaled corticosteroids and long-term asthma control in young children with asthma.

In this observational study, 81 2-6-year-old asthmatic children, using inhaled corticosteroids, closely followed-up in a programme with extensive self-management training, were enrolled. Adherence was measured daily for 12 months using Smartinhaler (Nexus6 Ltd, Auckland, New Zealand) devices. Longterm asthma control was assessed by parents and physicians and included clinical assessment, an asthma control questionnaire and lung function measurement. We examined the association of adherence to asthma control, adjusting for seasonal influences and clinical characteristics.

Median (interquartile range) adherence was 87\% (70-94\%), and 64 (79\%) children had well-controlled asthma throughout follow-up. Adherence $>80 \%$ was associated with better asthma control, and we found no important confounders of this association. Children with persistent mild symptoms had lower adherence rates $(\mathrm{p}=0.028)$.

Guideline-based asthma care was associated with good asthma control in most children. Adherence to inhaled corticosteroids was an independent strong predictor of long-term asthma control, with highest levels of asthma control found in children with adherence $>80 \%$ of doses prescribed.

@ERSpublications

Adherence to inhaled corticosteroids strongly predicts long-term asthma control in young children http://ow.ly/soc3S

Received: March 262013 | Accepted after revision: June 102013 | First published online: July 112013

Support statement: This study was supported by a grant from the Netherlands Asthma Foundation (grant no 3.4.06.007) and from the Foundation to Combat Asthma (Stichting Astmabestrijding).

Conflict of interest: Disclosures can be found alongside the online version of this article at www.erj.ersjournals.com 


\section{Introduction}

Daily low-dose inhaled corticosteroid (ICS) therapy significantly improves asthma control, both in schoolaged and in pre-school children with persistent asthma $[1,2]$. Poor adherence to therapy decreases the effectiveness of ICS, but studies of the relationship between adherence and asthma control are complicated by the different methods used to assess adherence. Electronic monitoring devices recording date and time of each inhaler actuation have been shown to be the most accurate measurement of adherence [3-5]. The few studies using this methodology in children showed an increased risk of uncontrolled asthma or an asthma exacerbation in children with lower adherence over the preceding 1- or 3-month period, respectively $[6,7]$. Although these studies therefore show an association between adherence and asthma control at a group level over short periods of time, no studies to date have examined the relationship between long-term asthma control and adherence in individual patients. A number of factors may influence this relationship over longer periods of follow-up. First, short-term adherence is likely to increase when parents know that adherence is being monitored, or when a follow-up visit is approaching, while long-term adherence decreases over time $[6,8]$. Secondly, individual characteristics such as significant comorbidity and seasonal changes in asthma control may influence the relationship of long-term asthma control and adherence [9]. Thirdly, the focus of guideline-based comprehensive asthma care is not only on adherence, but also on improving inhalation technique, elimination of exposure to relevant allergens and irritants (including environmental tobacco smoke) and treating comorbidities such as allergic rhinitis, all of which may contribute to improved asthma control [10]. Previous studies have shown that such comprehensive asthma care with regular follow-up was associated with achieving and maintaining well-controlled asthma in innercity asthmatic children [11, 12]. Although the authors suggested that adherence may explain the relationship between comprehensive asthma care and asthma control, adherence in these studies was assessed by healthcare providers instead of being monitored electronically.

We previously showed very high median electronically measured adherence rates and a significant association between adherence and asthma control in 2-6-year-old children with persistent asthma followed-up at our hospital-based paediatric asthma clinic (Princess Amalia Children's Clinic, Isala Klinieken, Zwolle, the Netherlands) for 3 months [13]. In the present study, we examined the association between daily electronically measured adherence and asthma control during 12 months of follow-up, which allowed us to take several potential confounders into account.

\section{Methods}

\section{Design and setting}

This was an observational study with 1-year follow-up of asthmatic children aged 2-6 years, who were being monitored and followed-up at our hospital-based paediatric asthma outpatient clinic. To enter our asthma management programme, children must be referred by their primary care practitioner because of troublesome, severe or frequent symptoms. After the diagnosis of persistent asthma had been made by the attending paediatrician, ICS by metered dose inhaler/spacer combination were prescribed as daily controller therapy, irrespective of the wheezing phenotype (episodic viral or multiple trigger wheeze), in accordance with the national paediatric asthma guidelines [14]. Details of the asthma management programme in our clinic have been published previously [13, 15], and comprise extensive training of correct inhalation technique, home visits to assess and reduce exposure to relevant allergens and tobacco smoke, and treatment of relevant comorbidities.

\section{Inclusion and collection of baseline data}

For this study, parents of all children aged 2-6 years with persistent asthma currently treated with ICS attending the outpatient clinic for regular follow-up were asked to participate in the study. Exclusion criteria comprised limited knowledge of the Dutch language and severe comorbidity. All included children whose ICS were withdrawn on a physician's advice during or immediately after completion of the study were excluded from analysis, because asthma control in these children was considered to be unrepresentative because of clinical asthma remission. We collected demographic and clinical data by structured interview and chart review. At baseline, lung function, asthma control and parental quality of life were also recorded. Lung function was assessed before and after inhaling salbutamol $400 \mu \mathrm{g}$ : flow-volume curves in children aged $\geqslant 5$ years, and respiratory resistance measured by the interrupter technique (Rint) using Microrint (Micro Medical Ltd, Rochester, UK) in children aged $<5$ years, according to European Respiratory Society/American Thoracic Society guidelines [16]. Results were expressed as Z-scores. Asthma control was assessed by parent-completed Asthma Control Questionnaire (ACQ) and parental quality of life by the Paediatric Asthma Caregiver Quality of Life questionnaire (PACQOL) [17, 18]. 
Assessment of adherence to ICS and follow-up

Throughout the 12-month follow-up, adherence was monitored by Smartinhaler (Nexus6 Ltd, Auckland, New Zealand), a validated electronic device logging the date and time of each ICS actuation [13, 19]. At each follow-up visit to the clinic, the data recorded by the Smartinhaler were uploaded by the primary investigator (who was not involved in the clinical care of these patients) who also checked proper recording function. The uploaded adherence rates were not available to the treating physicians, asthma nurses, parents or children. To prevent considerable loss of data in case of malfunctioning devices and to assess asthma control regularly, a home visit was made by a researcher to upload the Smartinhaler and record parental assessment of asthma control, when time to the next scheduled follow-up visit exceeded 5 months. We aimed at obtaining a minimum of four visits per patient during the study period. Because the follow-up ended with a visit to the outpatient clinic, and because these visits were mainly planned for regular asthma care, the study period exceeded 12 months in most patients.

Assessment of asthma control, lung function and parental quality of life during follow-up At each follow-up visit, asthma control was assessed by the attending physician and paediatric asthma nurse on a visual analogue scale ranging from 0 (completely uncontrolled asthma) to 10 (completely controlled asthma), based on a review of symptoms, limitations and exacerbations since the previous visit [20]. The prescribed daily dose and dosing frequency of maintenance treatment for asthma and any identified comorbid disorder was documented in the patient's chart at each follow-up visit, and recorded for study purposes. At each visit, parents completed an ACQ about their child, and reported any exacerbations that had occurred since the previous visit. After 6 and 12 months of follow-up, parents also completed the PACQOL, lung function was performed as described above, and a single-breath exhaled nitric oxide fraction ( FeNO) measurement was preformed using a hand-held electrochemical analyser (NIOX Mino; Aerocrine, Solna, Sweden) with an expiration time of $6 \mathrm{~s}$ [21]. After completion of the study, hospital and family physician charts were reviewed to double-check the reliability of the data, and to identify any prescriptions of oral prednisolone during the study period.

\section{Analysis}

Adherence was calculated as the number of Smartinhaler-recorded inhaled doses expressed as a percentage of the number of doses prescribed, either between midnight and midday or between midday and midnight for morning and evening doses, respectively, or at any time during the day for once-daily dosing. Adherence was censored at $100 \%$ of the prescribed dose. We assessed the association of adherence during the 2 months preceding each follow-up visit to each indicator of asthma control at the follow-up visit, using nonparametric methods because of the highly skewed distribution of adherence.

Because a number of children used a lower ICS dose, or no ICS at all, during summertime, and because we expected asthma control to deteriorate during winter months because of viral infections, we performed separated analyses for summer (May to September) and winter (October to April) seasons, expecting the relationship of adherence to asthma control to be stronger in winter. To compare the effects of moderate and good long-term adherence on asthma control, we also assessed the association of asthma control to 12 -month adherence dichotomised as good $(>80 \%)$ and moderate $(50-80 \%)$ adherence.

Based on all the collected information about asthma control, the child's long-term asthma control was classified as "well-controlled asthma" (no or infrequent mild symptoms during the study period), "mostly well-controlled asthma" (well controlled asthma, except for one or two episodes with moderate to severe symptoms sometimes requiring a course of prednisolone), "mild uncontrolled asthma" (recurrent periods with mild to moderate asthma symptoms), and "uncontrolled asthma" (recurrent periods with moderate or severe asthma symptoms including one or more exacerbations needing prednisolone).

Correlations between asthma control and clinical and demographic variables were assessed using Spearman's rank correlation coefficient. Because this yielded no statistically significant correlations, we refrained from multivariable analyses between adherence and asthma control, adjusting for other clinical and demographic variables.

\section{Ethical considerations}

This study was approved by the hospital ethics review board (Isala Klinieken) and all parents provided written informed consent.

\section{Results}

Of the 138 eligible patients, 104 (75\%) children entered the study after informed consent was obtained, and 81 (78\% of those enrolled) children with persistent prescriptions of ICS completed the 12-month follow-up (fig. 1). The clinical and demographic characteristics of these patients are presented in table 1. Most 


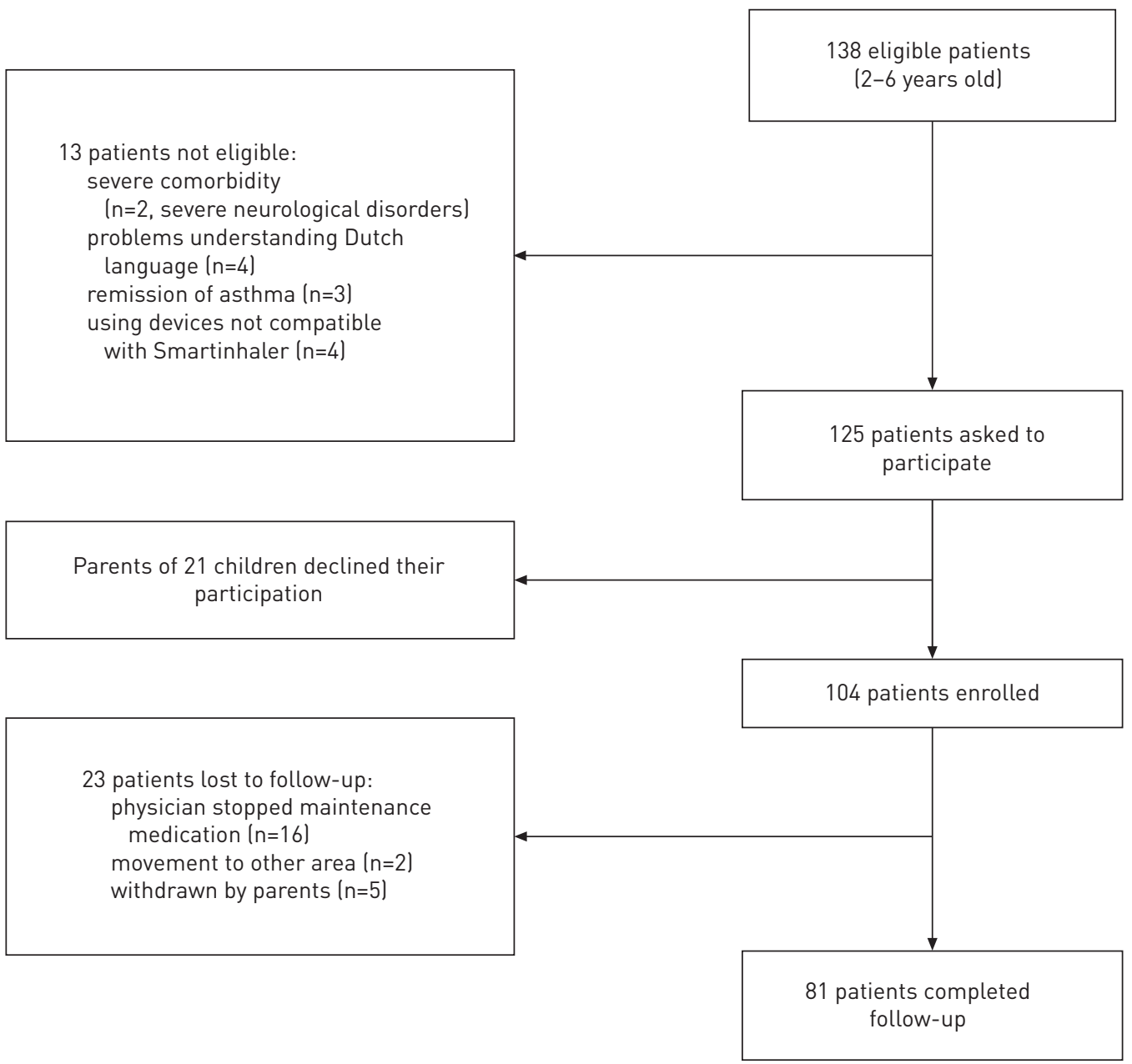

FIGURE 1 Inclusion of patients. Smartinhaler is manufactured by Nexus6 Ltd (Auckland, New Zealand).

participating children had well-controlled asthma using only low-dose ICS maintenance therapy. Reliable and reproducible lung function results were recorded in 60 (74\%) patients and inhalant allergen sensitisation results were available in 77 (95\%) patients (table 1). Electronic adherence data were collected for a median (interquartile range (IQR)) of 356 (286-388) days. Reasons for missing data included technical failure of the electronic monitoring devices, parents not bringing back the devices or bringing back damaged devices, and parents failing to use the device (e.g. leaving the electronic device at home during vacations).

The frequency distribution of adherence to daily ICS is presented in figure 2. Median (IQR) adherence was $87 \%(70-94 \%) .51(63 \%)$ children had high adherence $(>80 \%$ of prescribed dosages); adherence rates $<50 \%$ were recorded in only $10(12 \%)$ children (fig. 2$)$. The median (IQR) standard deviation of the monthly adherence rates for all 81 patients was $7.2 \%(4-11 \%)$, indicating that adherence was relatively constant in most patients.

Overall, asthma control during the 12-month study period was high and lung function was normal (table 2). Only two children were hospitalised because of an asthma exacerbation during the study period. The $15(19 \%)$ children who received a course of prednisolone during the study period had similar longterm adherence (median (IQR) 87\% (54-92\%)) to children not receiving prednisolone (median (IQR) $87 \%$ $(72-94 \%))(p=0.463)$. Exacerbations requiring prednisolone courses were not associated with low adherence in the 2 months preceding the exacerbation $(\mathrm{p}=0.552)$. However, in two children, exposure to lower ICS doses was associated with exacerbation occurrence: the exacerbation requiring prednisolone followed a physician-prescribed decrease of ICS dosage in one child and a pharmacy error (delivery of metered dose inhalers with lower ICS dose than prescribed) in the other. 
TABLE 1 Characteristics of study patients at baseline

\begin{tabular}{|c|c|}
\hline Subjects $\mathrm{n}$ & 81 \\
\hline Age years mean (range) & $4.6(2.2-6.9)$ \\
\hline Male & $51(63)$ \\
\hline Parental diagnosis of asthma \% & $33(41)$ \\
\hline Household smoking $\%$ & $24(30)$ \\
\hline Maternal educational level ${ }^{\#}$ & $5(4-7)$ \\
\hline Positive specific lgE to inhalant allergens & $43(56)$ \\
\hline Hospitalisation ever for asthma exacerbation & 40 (49) \\
\hline Duration of outpatient clinic asthma care before study months & $17(8-26)$ \\
\hline $\mathrm{FEV}_{1}{ }^{+} \mathrm{z}$-score & $0.67 \pm 1.1$ \\
\hline $\mathrm{FVC}^{+} \mathrm{z}$-score & $0.26 \pm 1.4$ \\
\hline $\operatorname{Rint}^{\S}$ z-score & $1.70 \pm 3.1$ \\
\hline $\mathbf{A C Q}{ }^{f}$ & $0.5(0.2-1.3)$ \\
\hline PACQOL $^{\#}$ & $6.2(5.3-6.8)$ \\
\hline \multicolumn{2}{|l|}{ Baseline medication } \\
\hline ICS & $73(90)$ \\
\hline ICS and long-acting bronchodilators \% & $8(10)$ \\
\hline ICS dose fluticasone $\mu \mathrm{g}$ mean (range) & $250(125-500)$ \\
\hline Nasal corticosteroids & $19(23)$ \\
\hline Oral antihistamine & 20 (25) \\
\hline
\end{tabular}

Data are presented as $\mathrm{n}(\%)$, median (interquartile range) or mean $\pm \mathrm{SD}$, unless otherwise stated. FEV1: forced expiratory volume in $1 \mathrm{~s}$; FVC: forced vital capacity; Rint: interruptor resistance; ACQ: Asthma Control Questionnaire; PACQOL: Paediatric Asthma Caregiver Quality of Life questionnaire; ICS: inhaled corticosteroid. \#: 1 (low) to 7 (high); ${ }^{\circledR}: \mathrm{n}=77 ;{ }^{+}: \mathrm{n}=33 ;^{\S}: \mathrm{n}=27{ }^{f}:<0.75$ (well controlled, $>1.5$ not well controlled).

The relationship of adherence with asthma control, lung function and FeNO values is presented in table 2. Adherence was associated with several indicators of asthma control, and asthma control was significantly better in children with good than in those with moderate adherence (table 2).

Against our expectations, the association between adherence and parent-assessed asthma control was stronger in summer than in winter (table 2). When completing the ACQ, many parents made remarks about having difficulties in attributing symptoms (cough) to viral respiratory tract infections or to asthma; this may have interfered with the association between adherence and asthma control. Children with high adherence had better spirometry results and lower FeNO levels, but no association was found between adherence and Rint results (table 2). In table 3, adherence levels in different categories of asthma control are compared. Four patients had severe uncontrolled asthma, three of whom had very high adherence to ICS/ long-acting bronchodilator combination therapy, along with a prescription of nasal steroids and antihistamines for comorbid allergic rhinitis. Compared with patients with (mostly) well-controlled asthma, children with mild uncontrolled asthma had significantly lower adherence rates (95\% CI of difference in median adherence rates $3-28 \%, \mathrm{p}=0.008)$, and mild uncontrolled asthma was rare among children with good adherence levels (fig. 3). Good adherence was accompanied by well-controlled asthma in $67 \%$ of the children, but was not associated with a lower likelihood of mild uncontrolled asthma or uncontrolled asthma (fig. 3a). To adjust the relationship between adherence and asthma control for the potential confounding by overtreatment of mild episodic asthma symptoms or asthma in clinical remission, we repeated this analysis, taking into account only those patients who were still using ICS 2 years after completion of the 12-month follow-up of adherence. The results are presented in figure 3b. There was a clear trend of higher levels of asthma control with higher levels of adherence $(\mathrm{p}=0.032)$, and patients with moderate levels of adherence (50-80\%) were significantly more likely to have mild uncontrolled asthma $(\mathrm{p}=0.028)$. Of all other clinical and demographic characteristics, only inhaled allergen sensitisation showed a trend to an association with good asthma control, although it did not reach statistical significance $(\mathrm{p}=0.075)$, and sensitisation prevalence was comparable in adherent $(>80 \%)$ and nonadherent $(<80 \%)$ patients. Therefore, no other confounders of the relationship of adherence and asthma control were identified.

\section{Discussion}

This study demonstrates the key role of adherence to ICS in achieving and maintaining asthma control in young children. In the large majority $(79 \%)$ of these patients, asthma could be well controlled most of the time over 12 months of guideline-based comprehensive asthma care. Only four (5\%) children had uncontrolled asthma, one of which had poor adherence to ICS. In comparison to earlier studies of long-term 


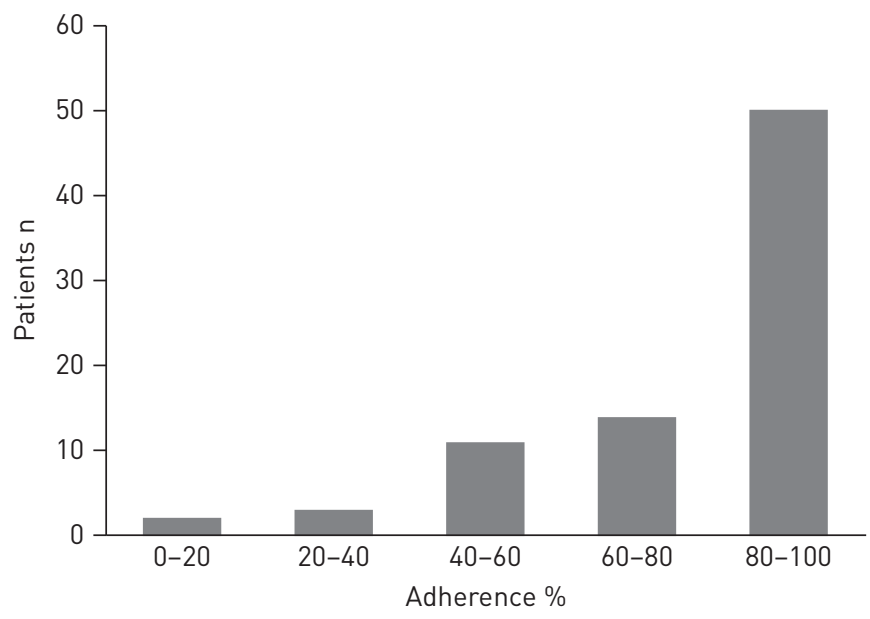

FIGURE 2 Electronically measured adherence to inhaled corticosteroids. $\mathrm{n}=81$.

adherence in childhood asthma $[6,22]$, median adherence was remarkably high $(87 \%)$ in our study cohort. Despite the overall high levels and associated limited variability of both adherence and asthma control, a significant and consistent association was found between the two variables. Asthma control was significantly better in children with good $(>80 \%)$ than in those with moderate (50-80\%) adherence (table 2 and fig. 3), and this association was independent of clinical and demographic characteristics. In particular, children with persistent mild asthma symptoms had lower adherence rates (table 3). Exacerbations requiring oral prednisolone and mild intermittent asthma symptoms were not associated with poor adherence in this cohort with overall high adherence and good asthma control.

Previous studies have also shown a high prevalence of well-controlled asthma during comprehensive guideline-based asthma care $[11,12,23]$. Because such comprehensive care comprises several components

TABLE 2 Indicators of asthma control and their association with electronically measured adherence during the 2 months before assessment of asthma control, and with 12-month adherence

Number of tests $\quad$ Test results
total/adherence/
comparison

\section{Adherence in the 2 months before clinic visit}

\section{Comparison between children with good ${ }^{\#}$ and moderate}

12-month adherence

Rank correlation
coefficient

p-value

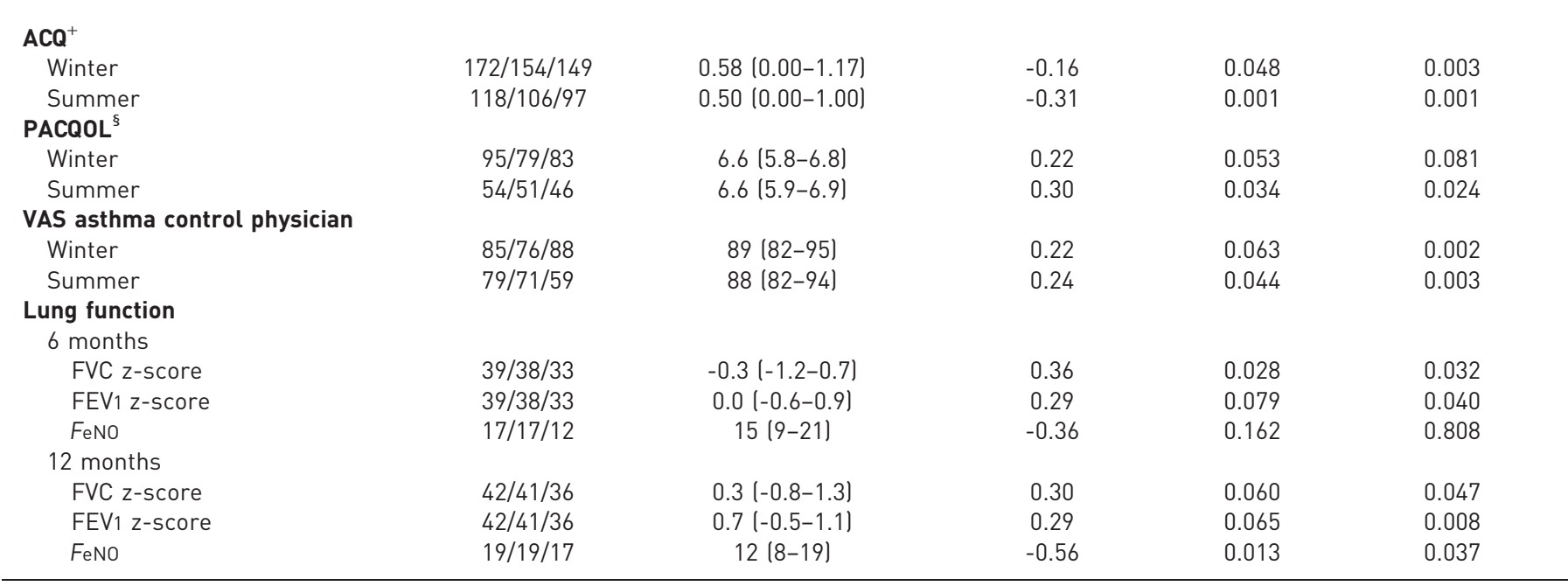

Data are presented as $\mathrm{n}$ or median (interquartile range), unless otherwise stated. ACQ: Asthma Control Questionnaire; PACQOL: Paediatric Asthma Caregiver Quality of Life questionnaire; VAS: visual analogue scale; FVC: forced vital capacity; FEV1: forced expiratory volume in $1 \mathrm{~s}$; FeNO: exhaled nitric oxide fraction. ${ }^{\#}:>80 \%$ adherence, $\mathrm{n}=51 ;{ }^{\uparrow}: 50-80 \%$ adherence, $\mathrm{n}=20 ;^{+}:<0.75$ (well controlled), $>1.5$ (not well controlled); ${ }^{\S}: 1$ (low) to 7 (high). 
TABLE 3 Level of long-term asthma control in the study population

Subjects $\quad$ Adherence $\%$

Difference versus wellcontrolled asthma p-value

\section{Well-controlled asthma Mostly well-controlled asthma Mild uncontrolled asthma Uncontrolled asthma}

$\begin{array}{cl}49(60) & 88(72-94) \\ 15(19) & 88(57-92) \\ 13(16) & 74(53-84) \\ 4(5) & 95(70-98)\end{array}$

Data are presented as $\mathrm{n}(\%)$ or median (interquartile range), unless otherwise stated. Asthma control was based on parental reports, physician's assessment and chart review.

that may promote asthma control, including reduction of allergen and irritant exposure, identification and treatment of relevant comorbidity, and ensuring proper inhalation technique and self-management, the independent role of adherence in determining asthma control remained uncertain in these studies. Our finding of a significant association of electronically measured adherence to both short-term and long-term asthma control, including lung function, in this population of young asthmatic children, independent of treatment of comorbidity and other clinical characteristics, shows that adherence to ICS plays a key role in achieving and maintaining asthma control.

In previous studies, substantially different levels of adherence needed to achieve asthma control were reported, ranging from $40-60 \%$ to $>80 \%[6,22]$. However, in these studies, a high prevalence of children with short-term asthma control despite low adherence to ICS was observed, suggesting ICS overtreatment of mild intermittent symptoms or asthma in clinical remission. To adjust for this potential confounder, we reanalysed the relationship between adherence and asthma control in a subgroup of children from our study who were still using ICS for persistent asthma 2 years after completion of the present study (fig. 3b). The association between adherence and asthma control was stronger in patients with ongoing persistent asthma (fig. 3b) than in the whole population comprising both ongoing persistent asthma and asthma in clinical remission (fig. 3a). Therefore, clinical remission of asthma in young children may help to explain why asthma can be well controlled in this age group despite poor adherence. In this study, in children who need ICS maintenance treatment for prolonged periods of time, good asthma control was related to high levels of adherence $(>80 \%)$. This emphasises the pivotal role of optimal adherence in achieving asthma control in children with chronic persistent asthma.

International guidelines recommend stepping up maintenance therapy in case of insufficient asthma control in the preceding months [10]. Our results suggest that adherence should be considered as a potential cause of such reduced asthma control, in particular when parents report that their child has frequent mild asthmatic symptoms (table 3). In clinical practice, many physicians will not step up maintenance therapy after a short episode of uncontrolled asthma while children have well-controlled asthma most of the time, and our results suggest that this pattern is not a marker of poor adherence to ICS.
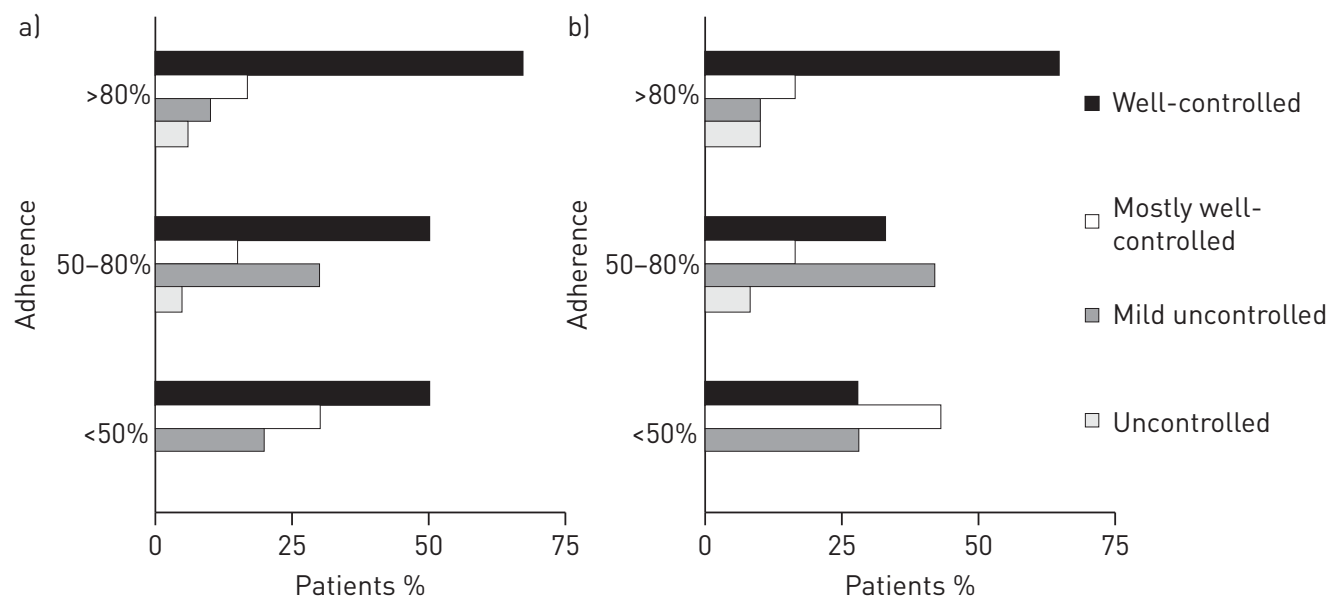

FIGURE 3 Relationship between asthma control and adherence in a) all patients $(\mathrm{n}=81)$ and $\mathrm{b})$ those patients in whom inhaled corticosteroids were continued for $\geqslant 2$ years after completing the 12 -month study period because of persistent asthma symptoms $(n=50)$. 


\section{Strengths and limitations}

The main strengths of our study include the longitudinal comprehensive assessment of asthma control and the objective, validated assessment of adherence over 12 months of follow-up. Asthma control is a multidimensional clinical construct which is not easily captured by a single questionnaire, particularly in children [24]. The assessment of asthma control by different methods in our study, using parental and physician assessment, validated questionnaires and lung function, is most likely to capture the clinical concept of asthma control described in asthma guidelines. Previous studies of the relationship between asthma control and adherence are hampered by a limited assessment of asthma control, use of less reliable methods of measuring adherence or short follow-up. Our long-term follow-up allowed us to analyse the influence of seasonal changes in adherence and asthma control, along with a range of potential clinical and demographic confounders, which enhances the robustness of the relationship between adherence and asthma control that we found.

The most important limitation of our study lies in its generalisability. Most parents and children in our study population came from Caucasian middle-class families, and the study was performed in a dedicated, secondary care, specialised asthma clinic. However, previous studies pointed out the importance of ICS adherence in determining good asthma control in inner-city populations from ethnic minorities [11, 12]. In addition, the relationship between adherence and asthma control in our study was not influenced by demographic and clinical characteristics of the study population. This suggests that adherence is a key factor in determining asthma control, irrespective of study setting and ethnic or social background of the population studied. Finally, the limited variability of asthma control and adherence in this study population may limit the study's power to identify determinants of either variable.

\section{Conclusions}

In most young children with asthma, well-controlled asthma can be achieved by comprehensive guidelinebased asthma care. The role of adherence to ICS in this relationship is crucial, because even in this highly adherent, well-controlled population, adherence was the only determinant significantly associated with asthma control, irrespective of other clinical or demographic characteristics. Persistent mild symptoms were a marker of suboptimal adherence. Adherence to ICS is a crucial factor to achieve and maintain asthma control in young children and should be a key focus of asthma care in this age group.

\section{References}

van Aalderen WM, Sprikkelman AB. Inhaled corticosteroids in childhood asthma: the story continues. Eur J Pediatr 2011; 170: 709-718.

2 Castro-Rodriguez JA, Pedersen S. The role of inhaled corticosteroids in management of asthma in infants and preschoolers. Curr Opin Pulm Med 2013; 19: 54-59.

3 Burgess SW, Sly PD, Morawska A, et al. Assessing adherence and factors associated with adherence in young children with asthma. Respirology 2008; 13: 559-563.

4 Ingerski LM, Hente EA, Modi AC, et al. Electronic measurement of medication adherence in pediatric chronic illness: a review of measures. J Pediatr 2011; 159: 528-534.

5 Jentzsch NS, Camargos PA, Colosimo EA, et al. Monitoring adherence to beclomethasone in asthmatic children and adolescents through four different methods. Allergy 2009; 64: 1458-1462.

6 Jentzsch NS, Camargos P, Sarinho ES, et al. Adherence rate to beclomethasone dipropionate and the level of asthma control. Respir Med 2012; 106: 338-343.

7 Milgrom H, Bender B, Ackerson L, et al. Noncompliance and treatment failure in children with asthma. J Allergy Clin Immunol 1996; 98: 1051-1057.

8 Modi AC, Pai AL, Hommel KA, et al. Pediatric self-management: a framework for research, practice, and policy. Pediatrics 2012; 129: e473-e485.

9 de Groot EP, Duiverman EJ, Brand PL. Comorbidities of asthma during childhood: possibly important, yet poorly studied. Eur Respir J 2010; 36: 671-678.

10 Global Initiative for Asthma (GINA). Global Strategy for Asthma Management and Prevention. www.ginasthma. org/documents/4 Date last accessed: January 15, 2012. Date last updated: December 2012.

11 Scott L, Morphew T, Bollinger ME, et al. Achieving and maintaining asthma control in inner-city children. J Allergy Clin Immunol 2011; 128: 56-63.

12 Jones CA, Clement LT, Morphew T, et al. Achieving and maintaining asthma control in an urban pediatric disease management program: the Breathmobile Program. J Allergy Clin Immunol 2007; 119: 1445-1453.

13 Klok T, Kaptein AA, Duiverman EJ, et al. High inhaled corticosteroids adherence in childhood asthma: the role of medication beliefs. Eur Respir J 2012; 40: 1149-1155.

14 Boluyt N, Rottier BL, de Jongste JC, et al. Assessment of controversial pediatric asthma management options using GRADE. Pediatrics 2012; 130: e658-e668.

15 Klok T, de Groot EP, Brouwer AF, et al. Follow-up of children with asthma. In: Carlsen KH, Gerritsen J, eds. Paediatric Asthma. Eur Respir Monogr 2012; 56: 210-223.

16 Beydon N, M'Buila C, Bados A, et al. Interrupter resistance short-term repeatability and bronchodilator response in preschool children. Respir Med 2007; 101: 2482-2487.

17 Juniper EF, Guyatt GH, Feeny DH, et al. Measuring quality of life in the parents of children with asthma. Qual Life Res 1996; 5: 27-34. 
18 Juniper EF, Bousquet J, Abetz L, et al. Identifying "well-controlled" and "not well-controlled" asthma using the Asthma Control Questionnaire. Respir Med 2006; 100: 616-621.

19 Burgess SW, Wilson SS, Cooper DM, et al. In vitro evaluation of an asthma dosing device: the smart-inhaler. Respir Med 2006; 100: 841-845.

20 Hammer SC, Robroeks CM, van Rij C, et al. Actual asthma control in a paediatric outpatient clinic population: do patients perceive their actual level of control? Pediatr Allergy Immunol 2008; 19: 626-633.

21 Koopman M, Arets HGM, Uiterwaal CS, et al. Comparing 6 and $10 \mathrm{sec}$ exhalation time in exhaled nitric oxide measurements in children. Pediatr Pulmomol 2009; 44: 340-344.

22 Lasmar L, Camargos P, Champs NS, et al. Adherence rate to inhaled corticosteroids and their impact on asthma control. Allergy 2009; 64: 784-789.

23 Gruchalla RS, Sampson HA, Matsui E, et al. Asthma morbidity among inner-city adolescents receiving guidelinesbased therapy: role of predictors in the setting of high adherence. J Allergy Clin Immunol 2009; 124: 213-221.

24 Cloutier MM, Schatz M, Castro M, et al. Asthma outcomes: composite scores of asthma control. J Allergy Clin Immunol 2012; 129: Suppl. 3, S24-S33. 